\title{
Actuator Fault Diagnosis for Discrete-Time Systems via Augmenting State Approach
}

\author{
Yongchao Wang, ${ }^{1,2}$ Shangmin Qi, ${ }^{1,2}$ Yujun Hu, ${ }^{1,2}$ Shenghui Guo $\mathbb{D}^{3}$, , and Darong Huang $\mathbb{D}^{4,}$ \\ ${ }^{1}$ Electric Power Research Institute, State Grid Xinjiang Electric Power Co., Ltd., Urumqi 830011, China \\ ${ }^{2}$ Marketing Service Center, State Grid Xinjiang Electric Power Co., Ltd., Urumqi 830011, China \\ ${ }^{3}$ College of Electronics and Information Engineering, Suzhou University of Science and Technology, Suzhou 215009, China \\ ${ }^{4}$ School of Information Science and Engineering, Chongqing Jiaotong University, Chongqing 400074, China \\ Correspondence should be addressed to Shenghui Guo; shguo@usts.edu.cn
}

Received 27 January 2021; Revised 9 April 2021; Accepted 3 May 2021; Published 25 May 2021

Academic Editor: Zhiwei Gao

Copyright () 2021 Yongchao Wang et al. This is an open access article distributed under the Creative Commons Attribution License, which permits unrestricted use, distribution, and reproduction in any medium, provided the original work is properly cited.

\begin{abstract}
For the problem of the actuator fault diagnosis in the control systems, this paper presents a novel method by using an interval estimation approach to detect the faults and reconstruct them. In order to make estimations of the unavoidable measurement noise, a descriptor system form is built. Firstly, a full-order interval observer is developed to detect actuator faults for its sensitiveness to them. Then, a reduced-order one, which is robust to actuator faults, is presented. This method does not need the boundary information of faults; thus, the design condition is more relaxed. In order to make the interval observer stable and cooperative, linear matrix inequalities and a time-varying transformation are employed to ensure the error system matrix to be Schur and nonnegative. Based on the interval estimation results of the aforementioned method, an interval reconstruction method of actuator faults is proposed. Finally, results of the two simulation examples verify the proposed methods are effective and accurate.
\end{abstract}

\section{Introduction}

Fault diagnosis (FD), including fault detection and isolation, is a very useful technique to improve the performances of control systems [1-5]. Many significant model-based FD methods have been developed, including, but not limited to, observer-based FD, filter-based FD, parity space, and parameter estimation. Among the abovementioned methods, the observer-based FD which is more practical and simpler, has become a powerful one with many results reported in the literature ([6-12] and references therein). Moreover, fault isolation technique is developed to locate the fault in the systems. Sometimes, the dynamic process of the fault and its actual value can even be obtained. Being the most powerful fault isolation technique, the fault reconstruction method has attracted much more attention than residual-based fault detection techniques ([2-4, 9, 13] and references therein). However, a number of methods in the literature are under some very restrictive assumptions which are impossible in many actual applications. For example, many FD results are proposed under the assumption that the disturbances or faults satisfy the well-known observer matching condition which is, in some sense, unrealistic for some practical systems. On the one hand, one of the major considerations in FD designs is how to deal with model uncertainties and external disturbances so that they cannot cause any negative effects on fault detection or fault reconstruction. On the other hand, it is not trivial to eliminate the negative impacts of model uncertainties or external disturbances on FD designs from the existing traditional observer-based FD methods. In fact, some FD design methods are developed, simply without considering model uncertainties and external disturbances. In recent two decades, interval observer has become popular for the systems with unknown inputs [14], and many process methods have been proposed [15-23]. The interval observer reveal only the over and under 
boundaries (denoted as $x_{k}^{+}$and $x_{k}^{-}$, respectively) of the system states $x_{k}$ instead of their asymptotic estimations. In comparison with the traditional observer design, it is much more convenient to construct an interval observer because many details about noises, disturbances, and nonlinear terms can be ignored, which is crucial in traditional observer design $[4,9,24]$. As we have known, a few researchers have investigated FD problems based on interval observers [25-32].

Considering the above background, our purpose is using interval observers to solve FD problems by constructing both of full-order and robust reduced-order interval observers. First, to eliminate the influence of measurement noise or disturbance, an augmented state method which was proposed by $[11,12]$ is adopted, viewing the measurement noise or disturbance as a system state, and then build a singular/descriptor system. Second, the observers are constructed and, based on the estimated results of the reducedorder observer, a practical fault reconstruction method is also proposed. The methods in the paper can be used in both of linear and nonlinear systems, such as electrical power systems, robot manipulator systems, and inverted pendulum systems. The major contributions of this work can be summarized as follows: (i) taking the inevitable measurement noise into account, an interval-observer-based fault detection means, which depend on the system's actual outputs and interval output estimates, are established; (ii) a reduced-order robust interval observer is developed for the equivalent system which is augmented; (iii) an interval reconstruction method of actuator faults is proposed based on the robust observer.

The paper is organized as follows. In Section 2, some basic definitions, background knowledge, and problem statements are introduced. Section 3 proposes a full-order interval observer and an actuator fault detection method. Section 4 designs a robust reduced-order interval observer, and an actuator fault interval reconstruction method is presented based on this. The correctness and effectiveness of the developed methods are shown through two simulation examples in Section 5. The conclusion is drawn in Section 6.

Some necessary notations marked in this paper are defined here. All of the inequality between two vectors $x, y \in \mathbf{R}^{n}$ or matrices $A, B \in \mathbf{R}^{m \times n}$ should be understood elementwise. For a constant matrix $A \in \mathbf{R}^{m \times n}$, define $A^{+}=$ $\max \{A, 0\}$ and $A^{-}=\max \{-A, 0\}$, so $A^{+} \geq 0, A^{-} \geq 0$, and $A=A^{+}-A^{-}$. For a square matrix $A \in \mathbf{R}^{n \times n}, A<0$ means that $A$ is a symmetric negative definite matrix. A matrix $A \in \mathbf{R}^{n \times n}$ is called a Schur matrix if its spectral radius is less than one and called a nonnegative matrix if all its elements are nonnegative.

\section{Preliminaries and Problem Statements}

Considering the following linear time-invariant discretetime system subjected to fault and outside disturbances,

$$
\left\{\begin{array}{l}
x_{k+1}=A x_{k}+B u_{k}+G f_{k}+D \eta_{k}, \\
y_{k}=C x_{k}+F \omega_{k},
\end{array}\right.
$$

where $x_{k} \in \mathbf{R}^{n_{x}}$ is the system state vector, $u_{k} \in \mathbf{R}^{n_{u}}$ is the control input vector, $y_{k} \in \mathbf{R}^{n_{y}}$ is the measurable output vector, $f_{k} \in \mathbf{R}^{n_{f}}$ and $\eta_{k} \in \mathbf{R}^{n_{\eta}}$ are the bounded actuator fault vector and the unknown input vector respectively, and $\omega_{k} \in \mathbf{R}^{n_{\omega}}$ is the measurement noise vector. All the parameter matrices are assumed with appropriate dimensions. The unknown input $\eta_{k} \in \mathbf{R}^{n_{\eta}}$, which is the combination of model uncertainties and outside disturbances, is bounded by known boundaries $\eta^{-} \in \mathbf{R}^{n_{\eta}}$ and $\eta^{+} \in \mathbf{R}^{n_{\eta}}$. Without loss of generality, we assume that $\operatorname{rank}(G)=n_{f}$ and $\operatorname{rank}(F)=n_{\omega}$. For the goal of estimating the measurement noise $\omega_{k}$, an augmented state method which was proposed in $[11,12]$ is used and new parameter matrices can be built as follows.

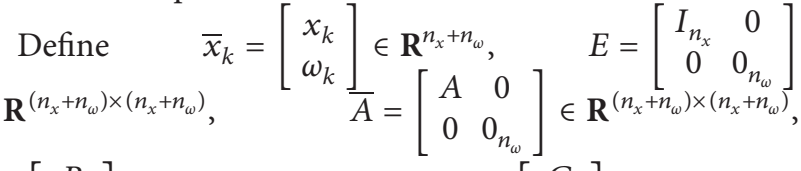

$$
\begin{aligned}
& \bar{B}=\left[\begin{array}{c}
B \\
0_{n_{\omega}}
\end{array}\right] \in \mathbf{R}^{\left(n_{x}+n_{\omega}\right) \times n_{u}}, \quad \bar{G}=\left[\begin{array}{c}
G \\
0_{n_{\omega}}
\end{array}\right] \in \mathbf{R}^{\left(n_{x}+n_{\omega}\right) \times n_{f}}, \\
& \bar{D}=\left[\begin{array}{c}
D \\
0_{n_{\omega}}
\end{array}\right] \in \mathbf{R}^{\left(n_{x}+n_{\omega}\right) \times n_{\eta}} \text {, and } \bar{C}=\left[\begin{array}{ll}
C & F
\end{array}\right] \text {. }
\end{aligned}
$$
form:

$$
\left\{\begin{array}{l}
E \bar{x}_{k+1}=\bar{A} \bar{x}_{k}+\bar{B} u_{k}+\bar{G} f_{k}+\bar{D} \eta_{k}, \\
y_{k}=\bar{C} \bar{x}_{k} .
\end{array}\right.
$$

Assuming that system (2) is detectable, that is, $\operatorname{rank}\left[\begin{array}{c}E \\ \bar{C}\end{array}\right]=n_{x}+n_{\omega}, \quad$ and $\quad \operatorname{rank}\left[\begin{array}{c}z E-\bar{A} \\ \bar{C}\end{array}\right]=n_{x}+n_{\omega}$, $\forall z \in \mathbf{C},\|z\| \geq 1$. We also assume that the initial condition $\bar{x}_{0}$ is bounded by two known vectors $\bar{x}_{0}^{-} \in \mathbf{R}^{n_{x}+n_{\omega}}$ and $\bar{x}_{0}^{+} \in \mathbf{R}^{n_{x}+n_{\omega}}$. It is obvious that

$$
\operatorname{rank}\left[\begin{array}{c}
E \\
\bar{C}
\end{array}\right]=\operatorname{rank}\left[\begin{array}{cc}
I_{n_{x}} & 0 \\
0 & 0_{n_{\omega}} \\
C & F
\end{array}\right]=n_{x}+n_{\omega} .
$$

So, there exists a full rank matrix $H_{1} \in \mathbf{R}^{\left(n_{x}+n_{\omega}\right) \times\left(n_{x}+n_{\omega}\right)}$ and a matrix $H_{2} \in \mathbf{R}^{\left(n_{x}+n_{\omega}\right) \times n_{y}}$ so that

$$
\left[\begin{array}{ll}
H_{1} & H_{2}
\end{array}\right]\left[\begin{array}{l}
E \\
\bar{C}
\end{array}\right]=I_{n_{x}+n_{\omega}} \text {. }
$$

Then, we can obtain

$$
\left\{\begin{array}{l}
\bar{x}_{k+1}=H_{1} \bar{A} \bar{x}_{k}+H_{1} \bar{B} u_{k}+H_{1} \bar{G} f_{k}+H_{1} \bar{D} \eta_{k}+H_{2} y_{k+1}, \\
y_{k}=\bar{C} \bar{x}_{k},
\end{array}\right.
$$

from (2). Let $\vartheta_{k}=\bar{x}_{k}-H_{2} y_{k}$, and (5) becomes

$$
\left\{\begin{array}{l}
\vartheta_{k+1}=H_{1} \bar{A} \vartheta_{k}+H_{1} \bar{A} H_{2} y_{k}+H_{1} \bar{B} u_{k}+H_{1} \bar{G} f_{k}+H_{1} \bar{D} \eta_{k}, \\
\left(I-\bar{C} H_{2}\right) y_{k}=\bar{C} \vartheta_{k} .
\end{array}\right.
$$

Lemma 1 (see [33]). For the following linear discrete-time system, 


$$
x_{k+1}=A x_{k}+d_{k} \text {, }
$$

if $A \in \mathbf{R}^{n_{x} \times n_{x}}$ is nonnegative, $d_{k} \in \mathbf{R}_{+}^{n_{x}}$, and the initial condition satisfies $x_{0} \geq 0$, then the system has nonnegative solutions $x_{k} \geq 0$ for $\forall k \geq 0$.

Lemma 2 (see [16]). For the discrete-time system,

$$
x_{k+1}=A x_{k},
$$

if $A \in \mathbf{R}^{n_{x} \times n_{x}}$ is a Schur constant matrix, then there exists a time-varying transformation $h_{k}=Q_{k} x_{k}$, which makes the system into a positive exponential stable system through such transformation, where $x_{k} \in \mathbf{R}^{n_{x}}$, the invertible matrices $Q_{k}$ satisfied $\left|Q_{k}\right|+\left|Q_{k}^{-1}\right| \leq c$ for arbitrary $k \geq 0$, and $c>0$ is a given nonnegative constant scalar.

Lemma 3 (see [15]). For any constant matrix $\zeta \in \mathbf{R}^{n_{u} \times n_{x}}$, if a vector variable $x_{k} \in \mathbf{R}^{n_{x}}$ satisfies $x_{k}^{-} \leq x_{k} \leq x_{k}^{+}$for some $x_{k}^{-}, x_{k}^{+} \in \mathbf{R}^{n_{x}}$, then one can obtain

$$
\zeta^{+} x_{k}^{-}-\zeta^{-} x_{k}^{+} \leq \zeta x_{k} \leq \zeta^{+} x_{k}^{+}-\zeta^{-} x_{k}^{-}
$$

\section{Actuator Fault Detection}

Generally speaking, the matrix $H_{1} \bar{A}$ is not Schur and nonnegative. For this reason, an equivalent state transformation $\sigma_{k}=T \vartheta_{k}$ is used, and system (6) can be converted into

$$
\left\{\begin{array}{l}
\sigma_{k+1}=\mathrm{TH}_{1} \mathrm{~T}^{-1} \sigma_{k}+\mathrm{TH}_{1} \mathrm{H} \bar{A}_{2} y_{k}+\mathrm{TH}_{1} \bar{B} u_{k}+\mathrm{TH}_{1} \bar{D} \eta_{k} \\
\left(I-\mathrm{H} \bar{C}_{2}\right) y_{k}=\mathrm{T}^{-1} \sigma_{k}
\end{array}\right.
$$

where $T \in \mathbf{R}^{\left(n_{x}+n_{\omega}\right) \times\left(n_{x}+n_{\omega}\right)}$ is an nonsingular matrix. The following interval observer can be designed:

$$
\left\{\begin{array}{l}
\sigma_{k+1}^{+}=T\left(H_{1} \bar{A}-\mathrm{L} \bar{C}\right) T^{-1} \sigma_{k}^{+}+\mathrm{TH}_{1} \mathrm{H} \bar{A}_{2} y_{k}+\mathrm{TL}\left(I-\mathrm{H}_{2}\right) y_{k}+\mathrm{TH}_{1} \bar{B} u_{k}+\left(\mathrm{TH}_{1} \bar{D}\right)^{+} \eta^{+}-\left(\mathrm{TH}_{1} \bar{D}\right)^{-} \eta^{-}, \\
\sigma_{k+1}^{-}=T\left(H_{1} \bar{A}-\mathrm{L} \bar{C}\right) T^{-1} \sigma_{k}^{-}+\mathrm{TH}_{1} \mathrm{H} \bar{A}_{2} y_{k}+\mathrm{TL}\left(I-\mathrm{H} \bar{C}_{2}\right) y_{k}+\mathrm{TH}_{1} \bar{B} u_{k}+\left(\mathrm{TH}_{1} \bar{D}\right)^{+} \eta^{-}-\left(\mathrm{TH}_{1} \bar{D}\right)^{-} \eta^{+}
\end{array}\right.
$$

where $L \in \mathbf{R}^{\left(n_{x}+n_{\omega}\right) \times n_{y}}$ is the observer gain matrix and $T$ and $L$ should be selected so that $T\left(H_{1} \bar{A}-\mathrm{L} \bar{C}\right) T^{-1}$ is a Schur matrix and a nonnegative matrix simultaneously. And, the nonsingular matrix $T$ and the gain matrix $L$ can be computed through the method which is described in Remark 1.
Theorem 1. Suppose that there is no actuator fault occurs. If we choose $\sigma_{0}^{+}=T^{+} \vartheta_{0}^{+}-T^{-} \vartheta_{0}^{-}$and $\sigma_{0}^{-}=T^{+} \vartheta_{0}^{-}-T^{-} \vartheta_{0}^{+}$, then system (11) is an interval observer for system (10).

Proof. Define the over and under errors as $\tilde{\sigma}_{k}^{+}=\sigma_{k}^{+}-\sigma_{k}$ and $\tilde{\sigma}_{k}^{-}=\sigma_{k}-\sigma_{k}^{-}$, respectively. From (10) and (11), we obtain

$$
\begin{aligned}
\tilde{\sigma}_{k+1}^{+}=\sigma_{k+1}^{+}-\sigma_{k+1} & =T\left(H_{1} \bar{A}-\mathrm{L} \bar{C}\right) T^{-1} \sigma_{k}^{+}-T\left(H_{1} \bar{A}-\mathrm{L} \bar{C}\right) T^{-1} \sigma_{k}+\left(\mathrm{TH}_{1} \bar{D}\right)^{+} \eta^{+}-\left(\mathrm{TH}_{1} \bar{D}\right)^{-} \eta^{-}-\left[\left(\mathrm{TH}_{1} \bar{D}\right)^{+}-\left(\mathrm{TH}_{1} \bar{D}\right)^{-}\right] \eta_{k} \\
& =T\left(H_{1} \bar{A}-\mathrm{L} \bar{C}\right) T^{-1} \tilde{\sigma}_{k}^{+}+\left(\mathrm{TH}_{1} \bar{D}\right)^{+}\left(\eta^{+}-\eta_{k}\right)+\left(\mathrm{TH}_{1} \bar{D}\right)^{-}\left(\eta_{k}-\eta^{-}\right), \\
\tilde{\sigma}_{k+1}^{-} & =T\left(H_{1} \bar{A}-\mathrm{L} \bar{C}\right) T^{-1} \tilde{\sigma}_{k}^{-}+\left(\mathrm{TH}_{1} \bar{D}\right)^{+}\left(\eta_{k}-\eta^{-}\right)+\left(\mathrm{TH}_{1} \bar{D}\right)^{-}\left(\eta^{+}-\eta_{k}\right) .
\end{aligned}
$$
so

Note that $\left(\mathrm{TH}_{1} \bar{D}\right)^{+} \geq 0,\left(T H_{1} \bar{D}\right)^{-} \geq 0$, and $\eta^{-} \leq \eta_{k} \leq \eta^{+}$,

$$
\begin{gathered}
\left(\mathrm{TH}_{1} \bar{D}\right)^{+}\left(\eta^{+}-\eta_{k}\right)+\left(\mathrm{TH}_{1} \bar{D}\right)^{-}\left(\eta_{k}-\eta^{-}\right) \geq 0, \\
\left(\mathrm{TH}_{1} \bar{D}\right)^{+}\left(\eta_{k}-\eta^{-}\right)+\left(\mathrm{TH}_{1} \bar{D}\right)^{-}\left(\eta^{+}-\eta_{k}\right) \geq 0 .
\end{gathered}
$$

Let $\sigma_{0}^{+}=T^{+} \vartheta_{0}^{+}-T^{-} \vartheta_{0}^{-}$and $\sigma_{0}^{-}=T^{+} \vartheta_{0}^{-}-T^{-} \vartheta_{0}^{+}$, and we have $\sigma_{0}^{-} \leq \sigma_{0} \leq \sigma_{0}^{+}$by using Lemma 3 . According to Lemma 1 , one can obtain that $\tilde{\sigma}_{k}^{+}=\sigma_{k}^{+}-\sigma_{k} \geq 0$ and $\tilde{\sigma}_{k}^{-}=\sigma_{k}-\sigma_{k}^{-} \geq 0$, i.e., $\sigma_{k} \leq \sigma_{k} \leq \sigma_{k}^{+}$, for arbitrary $k \geq 0$.

Remark 1. To find proper matrices $T$ and $L$, Raïssi et al. [18] propose a effective means to solve such problems. Because $T$ is a nonsingular matrix, one can construct a Sylvester equation from $\mathfrak{I}=T\left(H_{1} \bar{A}-\mathrm{L} \bar{C}\right) T^{-1}$ as follows:

$$
\begin{aligned}
\mathfrak{\Im} T+T\left(-H_{1} \bar{A}\right) & =-S \bar{C}, \\
S & =\mathrm{TL} .
\end{aligned}
$$

If the coefficient matrix $\left(-H_{1} \bar{A}\right)$ and the chosen matrix $\mathfrak{I}$ have no common eigenvalues, then (14) has a unique solution of $T$ and $L . S$ is an arbitrary matrix and $\mathfrak{I}$ can be chosen as a Schur and nonnegative diagonal matrix here.

Notice that $\bar{x}_{k}=\vartheta_{k}+H_{2} y_{k}=T^{-1} \sigma_{k}+H_{2} y_{k}$, the upper and lower estimations of $\bar{x}_{k}$ can also be given by 


$$
\left\{\bar{x}_{k}^{+}=\left(T^{-1}\right)^{+} \sigma_{k}^{+}-\left(T^{-1}\right)^{-} \sigma_{k}^{-}+H_{2} y_{k}, \bar{x}_{k}^{-}=\left(T^{-1}\right)^{+} \sigma_{k}^{-}-\left(T^{-1}\right)^{-} \sigma_{k}^{+}+H_{2} y_{k} .\right.
$$

Remark 2. We can also design the interval observer by a time-varying linear state transformation of $\sigma_{k}=T_{k} x_{k}$ rather than a constant one. In this way, a time-varying invertible matrix $T_{k}$ which can make $T_{k+1}\left(H_{1} \bar{A}-\mathrm{L} \bar{C}\right) T_{k}^{-1}$ into a Schur and nonnegative matrix can be found by using Lemma 2, where $L$ is calculated such that $\left(H_{1} \bar{A}-\mathrm{LC}\right)$ is a Schur matrix.

Then, the upper and lower estimation of $x_{k}, \omega_{k}$, and $y_{k}$ are $x_{k}^{+}=\left[\begin{array}{ll}I_{n_{x}} & 0_{n_{x} \times n_{\omega}}\end{array}\right] \bar{x}_{k}^{+}, x_{k}^{-}=\left[I_{n_{x}} 0_{n_{x} \times n_{\omega}}\right] \bar{x}_{k}^{-}, \omega_{k}^{+}=\left[0_{n_{\omega} \times n_{x}} I_{n_{\omega}}\right]$ $\bar{x}_{k}^{+}$, and $\omega_{k}^{-}=\left[\begin{array}{ll}0_{n_{\omega} \times n_{x}} & I_{n_{\omega}}\end{array}\right] \bar{x}_{k}^{-}$; then, the output will be limited by

$$
\left\{y_{k}^{+}=\bar{C}^{+} \bar{x}_{k}^{+}-\bar{C}^{-} \bar{x}_{k}^{-}, y_{k}^{-}=\bar{C}^{+} \bar{x}_{k}^{-}-\bar{C}^{-} \bar{x}_{k}^{+},\right.
$$

which can be a fault detector. That is, when the system runs without the presence of actuator faults, its output will meet $y_{k}^{-} \leq y_{k} \leq y_{k}^{+}$, otherwise the faults occur. It is noteworthy that since the interval is different from the artificially given threshold, the faults with small amplitudes may not all be detected. That is to say, for successful fault detection, the faults need to have enough amplitudes.

\section{Actuator Fault Interval Reconstruction}

For $\bar{C}=\left[\begin{array}{ll}C & F\end{array}\right]$, there exists an invertible matrix $N \in \mathbf{R}^{\left(n_{x}+n_{\omega}\right) \times\left(n_{x}+n_{\omega}\right)}$ such that

$$
\check{C}=\mathrm{N} \bar{C}=\left[\begin{array}{ll}
I_{n_{y}} & 0
\end{array}\right] .
$$

Then, system (6) can be transformed into

$$
\left\{\begin{array}{l}
\phi_{k+1}=N^{-1} H_{1} \mathrm{~N} \bar{A} \phi_{k}+N^{-1} H_{1} \mathrm{H} \bar{A}_{2} y_{k}+N^{-1} H_{1} \bar{B} u_{k}+N^{-1} H_{1} \bar{G} f_{k}+N^{-1} H_{1} \bar{D} \eta_{k}, \\
\left(I-\mathrm{H} \bar{C}_{2}\right) y_{k}=\mathrm{N} \bar{C} \phi_{k} .
\end{array}\right.
$$

Denote $\phi_{k}=N^{-1} \vartheta_{k}, \quad \check{A}=N^{-1} H_{1} \mathrm{~N} \bar{A}, \quad \check{B}=N^{-1} H_{1} \bar{B}$, $\check{G}=N^{-1} H_{1} \bar{G}, \check{D}=N^{-1} H_{1} \bar{D}$, and $\check{H}=N^{-1} H_{1} H \bar{A}_{2}$, and (18) becomes

$$
\left\{\begin{array}{l}
\phi_{k+1}=\check{A} \phi_{k}+\check{H} y_{k}+\check{B} u_{k}+\check{G} f_{k}+\check{D} \eta_{k}, \\
\left(I-\mathrm{H} \bar{C}_{2}\right) y_{k}=\check{C} \phi_{k} .
\end{array}\right.
$$

Decompose $\phi_{k}=\left[\begin{array}{ll}\phi_{1, k}^{\mathrm{T}} & \phi_{2, k}^{\mathrm{T}}\end{array}\right]^{\mathrm{T}}, \quad \phi_{1, k} \in \mathbf{R}^{n_{y}}$, and $\phi_{2, k}$ $\in \mathbf{R}^{n_{x}+n_{\omega}-n_{y}}$, we have

$$
\left(I-\mathrm{HC}_{2}\right) y_{k}=\left[\begin{array}{ll}
I_{n_{y}} & 0
\end{array}\right]\left[\begin{array}{c}
\phi_{1, k} \\
\phi_{2, k}
\end{array}\right]=\phi_{1, k}
$$

and the parameter matrices can be decomposed as

$$
\begin{aligned}
\check{A} & =\left[\begin{array}{ll}
\check{A}_{1} & \check{A}_{2} \\
\check{A}_{3} & \check{A}_{4}
\end{array}\right], \\
\check{B} & =\left[\begin{array}{l}
\check{B}_{1} \\
\check{B}_{2}
\end{array}\right], \\
\check{G} & =\left[\begin{array}{c}
\check{G}_{1} \\
\check{G}_{2}
\end{array}\right], \\
\check{D} & =\left[\begin{array}{c}
\check{D}_{1} \\
\check{D}_{2}
\end{array}\right], \\
\check{H} & =\left[\begin{array}{l}
\check{H}_{1} \\
\check{H}_{2}
\end{array}\right],
\end{aligned}
$$

where $\quad \check{A}_{1} \in \mathbf{R}^{n_{y} \times n_{y}}, \quad \check{B}_{1} \in \mathbf{R}^{n_{y} \times n_{u}}, \quad \check{G}_{1} \in \mathbf{R}^{n_{y} \times n_{f}}$, $\check{D}_{1} \in \mathbf{R}^{n_{y} \times n_{\eta}}$, and $\check{H}_{1} \in \mathbf{R}^{n_{y} \times n_{y}}$. Then, (22) can be easily obtained:

$$
\left\{\begin{array}{l}
\phi_{1, k+1}=\check{A}_{1} \phi_{1, k}+\check{A}_{2} \phi_{2, k}+\check{H}_{1} y_{k}+\check{B}_{1} u_{k}+\check{G}_{1} f_{k}+\check{D}_{1} \eta_{k} \\
\phi_{2, k+1}=\check{A}_{3} \phi_{1, k}+\check{A}_{4} \phi_{2, k}+\check{H}_{2} y_{k}+\check{B}_{2} u_{k}+\check{G}_{2} f_{k}+\check{D}_{2} \eta_{k} \\
\left(I-\mathrm{H} \bar{C}_{2}\right) y_{k}=\phi_{1, k},
\end{array}\right.
$$

and make an equivalent state transformation

$$
z_{k}=\left[\begin{array}{c}
z_{1, k} \\
z_{2, k}
\end{array}\right]=\mathcal{S} \phi(k)=\left[\begin{array}{cc}
I_{n_{y}} & 0 \\
\mathscr{L} & I_{n_{x}+n_{\omega}-n_{y}}
\end{array}\right]\left[\begin{array}{l}
\phi_{1, k} \\
\\
\phi_{2, k}
\end{array}\right],
$$

where $\mathscr{L} \in \mathbf{R}^{\left(n_{x}+n_{\omega}-n_{y}\right) \times n_{y}}$ is the gain matrix of the reducedorder observer, and we have $z_{1, k}=\phi_{1, k}=\left(I-\mathrm{H} \bar{C}_{2}\right) y_{k}$ and $z_{2, k}=\left[\begin{array}{ll}\mathscr{L} & I_{n_{x}+n_{\omega}-n_{y}}\end{array}\right] \phi_{k}$. So, $z_{2, k}$ is described by the following equation: 


$$
\begin{aligned}
z_{2, k+1} & =\left[\mathscr{L} I_{n_{x}+n_{\omega}-n_{y}}\right] \phi_{k+1}=\left(\check{A}_{4}+\mathscr{L} \check{A}_{2}\right) z_{2, k}+\left[\left(\check{A}_{3}+\mathscr{L} \check{A}_{1}\right)-\left(\check{A}_{4}+\mathscr{L} \check{A}_{2} \mathscr{L}\right](I-\mathrm{H} \bar{C}) y_{k}+\left(\mathscr{L} \check{H}_{1}+\check{H}_{2}\right) y_{k}\right. \\
& +\left(\mathscr{L} \check{B}_{1}+\check{B}_{2}\right) u_{k}+\left(\mathscr{L} \check{D}_{1}+\check{D}_{2}\right) \eta_{k}+\left(\mathscr{L} \check{G}_{1}+\check{G}_{2}\right) f_{k} .
\end{aligned}
$$

Lemma 4 (see [9]). If there exist a symmetric positive definite matrix $P \in \mathbf{R}^{\left(n_{x}+n_{\omega}-n_{y}\right) \times\left(n_{x}+n_{\omega}-n_{y}\right)}$ and a matrix $Q \in \mathbf{R}^{\left(n_{x}+n_{\omega}-n_{y}\right) \times n_{y}}$ for which the following LMIs

$$
\begin{gathered}
\min \delta \\
{\left[\begin{array}{cc}
-\delta I & {\left[\begin{array}{ll}
Q & P
\end{array}\right] \check{G} .} \\
\check{G}^{T}\left[\begin{array}{ll}
Q & P
\end{array}\right] & -\delta I
\end{array}\right] \prec 0,} \\
{\left[\begin{array}{cc}
-P & \check{A}_{4}^{T} P+\check{A}_{2}^{T} Q \\
P \check{A}_{4}+Q \check{A}_{2} & -P
\end{array}\right] \prec 0,}
\end{gathered}
$$

are solvable, then the observer gain $\mathscr{L}=P^{-1} \mathrm{Q}$ can make $\check{A}_{4}+$ $\mathscr{L} \check{A}_{2}$ into a Schur matrix, such that

$$
\mathscr{L} \check{G}_{1}+\check{G}_{2}=0 \text {. }
$$

If we denote $\mathscr{A}=\check{A}_{4}+\mathscr{L} \check{A}_{2}, \quad \aleph=\left[\left(\check{A}_{3}+\mathscr{L} \check{A}_{1}\right)-\right.$ $\left.\left(\check{A}_{4}+\mathscr{L} \check{A}_{2}\right) \mathscr{L}\right]\left(I-H \bar{C}_{2}\right)+\left(\mathscr{L} \check{H}_{1}+\check{H}_{2}\right), \quad \mathscr{B}=\mathscr{L} \check{B}_{1}+\check{B}_{2}$, and $\mathscr{D}=\mathscr{L} \check{D}_{1}+\check{D}_{2}$, and notice (27); then, (24) becomes

$$
z_{2, k+1}=\mathscr{A} z_{2, k}+\aleph y_{k}+\mathscr{B} u_{k}+\mathscr{D} \eta_{k} \text {. }
$$

When $\mathscr{A}$ is not a nonnegative matrix, a time-varying transformation $\xi_{k}=\mathscr{T}_{k} z_{2, k}$ can be used here to handle the problem, so we obtain

$$
\xi_{k+1}=\mathscr{T}_{k+1} \mathscr{A} \mathscr{T}_{k}^{-1} \xi_{k}+\mathscr{T}_{k+1} \aleph y_{k}+\mathscr{T}_{k+1} \mathscr{B} u_{k}+\mathscr{T}_{k+1} \mathscr{D} \eta_{k} .
$$

From Lemma 2, it is obvious that a time-varying invertible matrix $\mathscr{T}_{k}$ can be obtained.

Then, an interval observer for (29) is constructed as

$$
\left\{\begin{array}{l}
\xi_{k+1}^{+}=\mathscr{T}_{k+1} \mathscr{A} \mathscr{T}_{k}^{-1} \xi_{k}^{+}+\mathscr{T}_{k+1} \aleph y_{k}+\mathscr{T}_{k+1} \mathscr{B} u_{k}+\left(\mathscr{T}_{k+1} \mathscr{D}\right)^{+} \eta^{+}-\left(\mathscr{T}_{k+1} \mathscr{D}\right)^{-} \eta^{-} \\
\xi_{k+1}^{-}=\mathscr{T}_{k+1} \mathscr{A} \mathscr{T}_{k}^{-1} \xi_{k}^{-}+\mathscr{T}_{k+1} \aleph y_{k}+\mathscr{T}_{k+1} \mathscr{B} u_{k}+\left(\mathscr{T}_{k+1} \mathscr{D}\right)^{+} \eta^{-}-\left(\mathscr{T}_{k+1} \mathscr{D}\right)^{-} \eta^{+}
\end{array}\right.
$$

Theorem 2. If LMIs (25) and (26) are solvable, let $\vartheta_{0}^{+}=\bar{x}_{0}^{+}-H_{2} y_{0}, \vartheta_{0}^{-}=\bar{x}_{0}^{-}-H_{2} y_{0}, \phi_{0}^{+}=\left(N^{-1}\right)^{+} \vartheta_{0}^{+}-\left(N^{-1}\right)^{-}$ $\vartheta_{0}^{-}, \phi_{0}^{-}=\left(N^{-1}\right)^{+} \vartheta_{0}^{-}-\left(N^{-1}\right)^{-} \vartheta_{0}^{+}$, and

$$
\left\{\begin{array}{l}
z_{2,0}^{+}=\left[\mathscr{L}^{+} I_{n_{x}+n_{\omega}-n_{y}}\right] \phi_{0}^{+}-\left[\mathscr{L}^{-} 0_{n_{x}+n_{\omega}-n_{y}}\right.
\end{array}\right] \phi_{0}^{-},\left\{\begin{array}{l}
\xi_{0}^{+}=\mathscr{T}_{0}^{+} z_{0}^{+}-\mathscr{T}_{0}^{-} z_{0}^{-}, \\
z_{2,0}^{-}=\left[\mathscr{L}^{+} I_{n_{x}+n_{\omega}-n_{y}}\right] \phi_{0}^{-}-\left[\mathscr{L}^{-} 0_{n_{x}+n_{\omega}-n_{y}}\right.
\end{array}\right] \phi_{0}^{+},\left\{\begin{array}{l}
\xi_{0}^{-}=\mathscr{T}_{0}^{+} z_{0}^{-}-\mathscr{T}_{0}^{-} z_{0}^{+} .
\end{array}\right.
$$

Then, the solutions of (29) and (30) satisfy $\xi_{k}^{-} \leq \xi_{k} \leq \xi_{k}^{+}$, for $\forall k \geq 0$. In other words, (30) is an interval observer of (29).

Proof. We notice that $\mathscr{T}_{k+1} \mathscr{A} \mathscr{T}_{k}^{-1}$ is Schur and nonnegative matrix, so one can refer to Theorem 1 and the proof is omitted here for brevity.

Next, an interval reconstruction method is proposed to reconstruct actuator faults. There exists a nonsingular matrix $M \in \mathbf{R}^{\left(n_{x}+n_{\omega}\right) \times\left(n_{x}+n_{\omega}\right)}$ such that

$$
\mathrm{MH}_{1} \bar{G}=\left[\begin{array}{c}
I_{n_{f}} \\
0_{n_{x}+n_{\omega}-n_{f}}
\end{array}\right] .
$$

Then, system (6) can be rewritten as

$$
\begin{aligned}
M \bar{\vartheta}_{k+1}= & \left(\mathrm{MH}_{1} \bar{A} M^{-1}\right) M \bar{\vartheta}_{k}+\mathrm{MH}_{1} \mathrm{H} \bar{A}_{2} y_{k}+\mathrm{MH}_{1} \bar{B} u_{k} \\
& +\mathrm{MH}_{1} \bar{G} f_{k}+\mathrm{MH}_{1} \bar{D} \eta_{k} .
\end{aligned}
$$

The matrices and vector can be decomposed as follows:

$$
\begin{aligned}
\mathrm{MH}_{1} \mathrm{M}^{-1} & =\left[\begin{array}{ll}
\widehat{A}_{1} & \widehat{A}_{2} \\
\widehat{A}_{3} & \widehat{A}_{4}
\end{array}\right], \\
\mathrm{MH}_{1} \mathrm{H} \bar{A}_{2} & =\left[\begin{array}{c}
\widehat{H}_{1} \\
\widehat{H}_{2}
\end{array}\right], \\
\mathrm{MH}_{1} \bar{B} & =\left[\begin{array}{l}
\widehat{B}_{1} \\
\widehat{B}_{2}
\end{array}\right], \\
\mathrm{MH}_{1} \bar{D} & =\left[\begin{array}{l}
\widehat{D}_{1} \\
\widehat{D}_{2}
\end{array}\right], \\
M \bar{\vartheta}_{k} & =\left[\begin{array}{l}
\widehat{\vartheta}_{1, k} \\
\widehat{\vartheta}_{2, k}
\end{array}\right],
\end{aligned}
$$


where $\widehat{A}_{1} \in \mathbf{R}^{n_{f} \times n_{f}}, \quad \widehat{A}_{2} \in \mathbf{R}^{n_{f} \times\left(n_{x}+n_{\omega}-n_{f}\right)}, \quad \widehat{H}_{1} \in \mathbf{R}^{n_{f} \times n_{y}}$, $\widehat{B}_{1} \in \mathbf{R}^{n_{f} \times n_{u}}, \widehat{D}_{1} \in \mathbf{R}^{n_{f} \times n_{\eta}}$, and $\widehat{\vartheta}_{1, k} \in \mathbf{R}^{n_{f}}$ and $\widehat{\vartheta}_{2, k} \in \mathbf{R}^{n_{x}+n_{\omega}-n_{f}}$; then, the dynamic for $\widehat{\vartheta}_{1, k}$ is governed by

$$
\widehat{\vartheta}_{1, k+1}=\widehat{A}_{1} \widehat{\vartheta}_{1, k}+\widehat{A}_{2} \widehat{\vartheta}_{2, k}+\widehat{H}_{1} y_{k}+\widehat{B}_{1} u_{k}+\widehat{D}_{1} \eta_{k}+f_{k} .
$$

Define

$$
\left\{\begin{array}{l}
\widehat{\vartheta}_{k}^{+}=M^{+} \vartheta_{k}^{+}-M^{-} \vartheta_{k}^{-}, \\
\widehat{\vartheta}_{k}^{-}=M^{+} \vartheta_{k}^{-}-M^{-} \vartheta_{k}^{+},
\end{array}\right.
$$

and then, $\widehat{\vartheta}_{k}^{+}$and $\widehat{\vartheta}_{k}^{-}$are the upper and lower interval estimations of $\widehat{\vartheta}_{k}$, respectively, i.e., we have $\widehat{\vartheta}_{k}^{-} \leq \widehat{\vartheta}_{k} \leq \widehat{\vartheta}_{k}^{+}$. Let

$$
\begin{aligned}
& \widehat{\vartheta}_{k}^{+}=\left[\begin{array}{l}
\hat{\vartheta}_{1, k}^{+} \\
\hat{\vartheta}_{2, k}^{+}
\end{array}\right], \\
& \widehat{\vartheta}_{k}^{-}=\left[\begin{array}{l}
\hat{\vartheta}_{1, k}^{-} \\
\widehat{\vartheta}_{2, k}^{-}
\end{array}\right],
\end{aligned}
$$

where $\widehat{\vartheta}_{1, k}^{+}, \widehat{\vartheta}_{1, k}^{-} \in \mathbf{R}^{n_{f}}$. And, we have

$$
\begin{aligned}
& \widehat{\vartheta}_{1, k}^{-} \leq \widehat{\vartheta}_{1, k} \leq \widehat{\vartheta}_{1, k}^{+}, \\
& \widehat{\vartheta}_{2, k}^{-} \leq \widehat{\vartheta}_{2, k} \leq \widehat{\vartheta}_{2, k}^{+} .
\end{aligned}
$$

Now, from (35), we obtain

$$
f_{k}=\widehat{\vartheta}_{1, k+1}-\widehat{A}_{1} \widehat{\vartheta}_{1, k}-\widehat{A}_{2} \widehat{\vartheta}_{2, k}-\widehat{H}_{1} y_{k}-\widehat{B}_{1} u_{k}-\widehat{D}_{1} \eta_{k} \text {. }
$$

From (39), the actuator fault interval reconstruction can be built as follows:

$$
\left\{\begin{array}{l}
f_{k}^{+}=\widehat{\vartheta}_{1, k+1}^{+}-\widehat{A}_{1}^{+} \widehat{\vartheta}_{1, k}^{-}+\widehat{A}_{1}^{-} \widehat{\vartheta}_{1, k}^{+}-\widehat{A}_{2}^{+} \widehat{\vartheta}_{2, k}^{-}+\widehat{A}_{2}^{-} \widehat{\vartheta}_{2, k}^{+}-\widehat{H}_{1} y_{k}-\widehat{B}_{1} u_{k}-\widehat{D}_{1}^{+} \eta^{-}+\widehat{D}_{1}^{-} \eta^{+}, \\
f_{k}^{-}=\widehat{\vartheta}_{1, k+1}^{-}-\widehat{A}_{1}^{+} \widehat{\vartheta}_{1, k}^{+}+\widehat{A}_{1}^{-} \widehat{\vartheta}_{1, k}^{-}-\widehat{A}_{2}^{+} \widehat{\vartheta}_{2, k}^{+}+\widehat{A}_{2}^{-} \widehat{\vartheta}_{2, k}^{-}-\widehat{H}_{1} y_{k}-\widehat{B}_{1} u_{k}-\widehat{D}_{1}^{+} \eta^{+}+\widehat{D}_{1}^{-} \eta^{-}
\end{array}\right.
$$

Theorem 3. For $f_{k}^{+}$and $f_{k}^{-}$defined by (40), we have $f_{k}^{-} \leq f_{k} \leq f_{k}^{+}$, for $\forall k \geq 0$.

Proof. Notice that $\widehat{\vartheta}_{1, k}^{-} \leq \widehat{\vartheta}_{1, k} \leq \widehat{\vartheta}_{1, k}^{+}, \quad \widehat{\vartheta}_{2, k}^{-} \leq \widehat{\vartheta}_{2, k} \leq \widehat{\vartheta}_{2, k}^{+}$, $\eta^{-} \leq \eta_{k} \leq \eta^{+}$, and $\widehat{A}_{i}=\widehat{A}_{i}^{+}-\widehat{A}_{i}^{-}(i=1,2)$, and we can verify that $f_{k}^{+}-f_{k} \geq 0$ and $f_{k}-f_{k}^{-} \geq 0$ hold for all $k \geq 0$ easily.

Remark 3. Generally speaking, the basic idea of an interval state observer design is to construct a couple of new systems which can produce interval estimations for the original system states by using the information of the boundaries of unknown inputs together with, if necessary, the information of the measured outputs of the original system. So, designing an interval state observer for the system with unknown inputs, the knowledge of the boundaries of the unknown inputs is usually crucial. In the present paper, on the one hand, with reduced-order observer design techniques, an interval reduced-order observer is developed without knowing the boundary information of the actuator faults, which can actually be regarded as unknown inputs. On the other hand, based on the partial known information, an interval reconstruction method of the actuator fault is developed.

\section{Numerical Simulation}

In this section, the details of the design process of the developed methods are given through a numerical example and a practical system; then, the effectiveness is illustrated.
5.1. Numerical Example. Consider a discrete-time system (1) and the parameter matrices are as follows:

$$
\begin{aligned}
A & =\left[\begin{array}{ccc}
0.1 & 0.4 & 0 \\
0.5 & 0.2 & 0.3 \\
0 & -0.2 & 0
\end{array}\right], \\
B & =\left[\begin{array}{l}
1 \\
1 \\
2
\end{array}\right], \\
G & =\left[\begin{array}{l}
2 \\
1 \\
1
\end{array}\right], \\
D & =\left[\begin{array}{l}
1 \\
1 \\
1
\end{array}\right], \\
C & =\left[\begin{array}{ll}
1 & 0 \\
0 & 1
\end{array}\right], \\
F & =\left[\begin{array}{l}
1.2 \\
1.5
\end{array}\right] .
\end{aligned}
$$

Then, the parameter matrices in the rewritten system (6) are presented below: 


$$
\begin{aligned}
& E=\left[\begin{array}{llll}
1 & 0 & 0 & 0 \\
0 & 1 & 0 & 0 \\
0 & 0 & 1 & 0 \\
0 & 0 & 0 & 0
\end{array}\right], \\
& \bar{A}=\left[\begin{array}{cccc}
0.1 & 0.4 & 0 & 0 \\
0.5 & 0.2 & 0.3 & 0 \\
0 & -0.2 & 0 & 0 \\
0 & 0 & 0 & 0
\end{array}\right] \text {, } \\
& \bar{B}=\left[\begin{array}{l}
1 \\
1 \\
2 \\
0
\end{array}\right] \text {, } \\
& \bar{C}=\left[\begin{array}{llll}
1 & 0 & 1 & 1.2 \\
0 & 1 & 1 & 1.5
\end{array}\right] \text {, } \\
& \bar{G}=\left[\begin{array}{l}
2 \\
1 \\
1 \\
0
\end{array}\right] \text {, } \\
& \bar{D}=\left[\begin{array}{l}
1 \\
1 \\
1 \\
0
\end{array}\right] \text {. }
\end{aligned}
$$

We assume that control input, unknown disturbances, and actuator fault are

$$
\begin{aligned}
u_{k} & =(3-0.03 k) \sin (0.25 k), \\
\eta_{k} & =0.1 \sin (0.15 k), \\
\omega_{k} & =0.4 \sin (0.5 k+0.5), \\
f_{k} & = \begin{cases}1.8(\sin (0.25 k)+\cos (0.3 k+1)), & 20 \leq k \leq 100, \\
0, & \text { else. }\end{cases}
\end{aligned}
$$

The selected initial conditions are

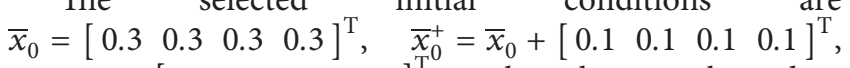
$\bar{x}_{0}^{-}=\bar{x}_{0}-\left[\begin{array}{llll}0.1 & 0.1 & 0.1 & 0.1\end{array}\right]^{\mathrm{T}}, \eta_{k}$ has the over boundary $\eta^{+}=0.1$, and the under boundary $\eta^{-}=-0.1$.
The matrix of $H_{1}$ and $H_{2}$ can be chosen as

$$
\begin{aligned}
& H_{1}=\left[\begin{array}{cccc}
1 & 0 & 0 & 0 \\
0 & 1 & 0 & 0 \\
0 & 0 & 1 & 0 \\
-0.2811 & -0.4418 & -0.7229 & 1
\end{array}\right], \\
& H_{2}=\left[\begin{array}{cc}
0 & 0 \\
0 & 0 \\
0 & 0 \\
0.2811 & 0.4418
\end{array}\right] .
\end{aligned}
$$

To construct a Sylvester equation, we choose that

$$
\begin{aligned}
& \mathfrak{J}=\left[\begin{array}{cccc}
0.41 & 0 & 0 & 0 \\
0 & 0.23 & 0 & 0 \\
0 & 0 & 0.61 & 0 \\
0 & 0 & 0 & 0.86
\end{array}\right], \\
& S=\left[\begin{array}{ll}
1 & 0 \\
0 & 1 \\
1 & 1 \\
1 & 0
\end{array}\right] .
\end{aligned}
$$

Then, substitute them into (14) and compute it, we have

$$
\begin{aligned}
T & =\left[\begin{array}{rrrr}
-1.5070 & -0.3918 & -1.7797 & -2.9268 \\
-1.3236 & -3.5919 & -5.2750 & -6.5217 \\
-1.5516 & -1.7869 & -3.1958 & -4.4262 \\
-0.9650 & -0.1617 & -1.0042 & -1.3953
\end{array}\right], \\
L & =\left[\begin{array}{cc}
-2.5867 & -2.1528 \\
-1.3643 & -3.3595 \\
0.5158 & 2.8671 \\
0.8592 & -0.1851
\end{array}\right] .
\end{aligned}
$$

Figure 1 shows the results of interval estimations when using full-order interval observer (11), and in the system, there are no actuator faults. The fault detection is plotted in Figure 2, and it reveals that the faults occur during the time period of $[20,100]$.

Next is the design process of the robust interval observer. The invertible matrix is 


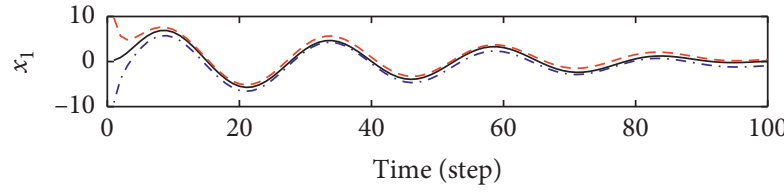

(a)

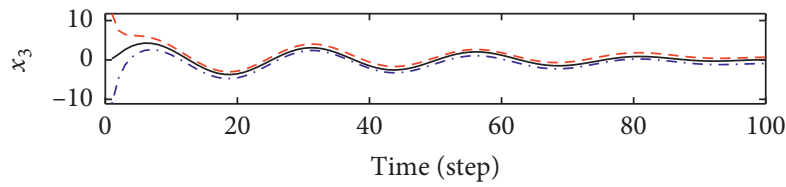

(c)

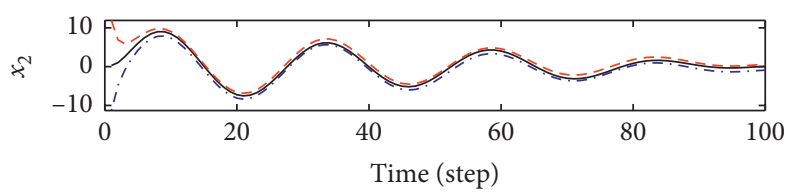

(b)

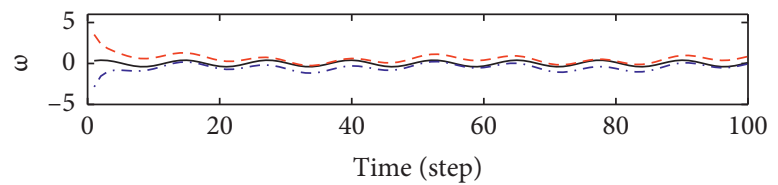

(d)

Figure 1: Interval estimation without actuator fault, i.e., $f_{k} \equiv 0$. Real state-black solid line; the upper boundary of estimations-red dash line; the lower boundary of estimations-blue dash dot line.

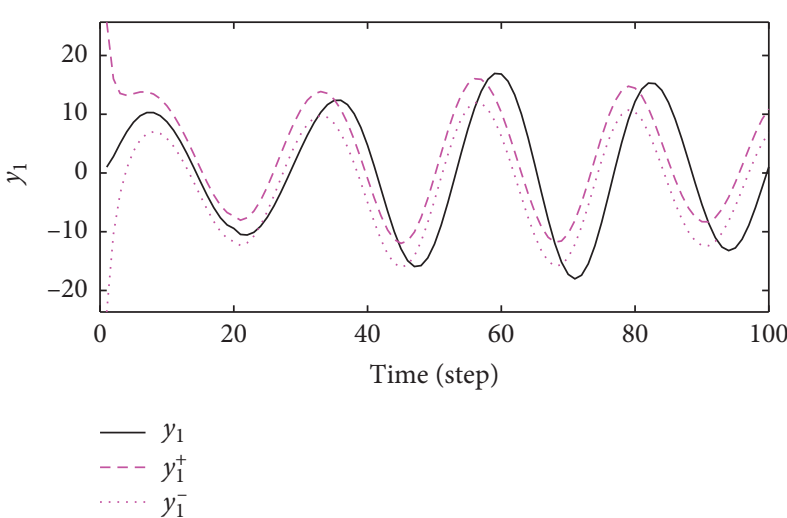

(a)

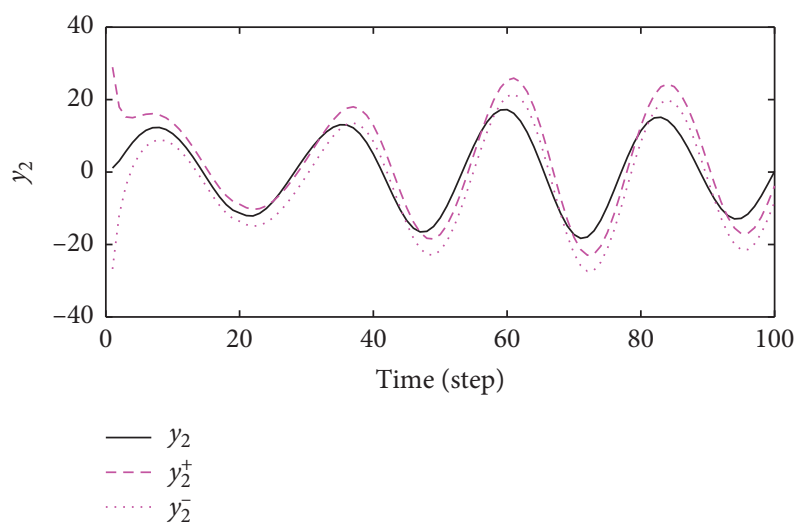

(b)

FIgURE 2: Fault detection.

$$
N=\left[\begin{array}{cccc}
0.4348 & -0.4348 & 0.1304 & 0.4348 \\
-0.4348 & 0.4348 & -0.1304 & 0.5652 \\
1.0870 & -0.0870 & -1.1739 & 0.0870 \\
-0.4348 & 0.4348 & 0.8696 & -0.4348
\end{array}\right]
$$

Compute LMIs (25) and (26), we have

$$
\begin{aligned}
& P=10^{5} \times\left[\begin{array}{cc}
8.8106 & -1.8701 \\
-1.8701 & 4.9109
\end{array}\right], \\
& Q=10^{6} \times\left[\begin{array}{ll}
0.7364 & 0.6144 \\
0.7612 & 3.6053
\end{array}\right], \\
& \mathscr{L}=\left[\begin{array}{ll}
1.2672 & 2.4539 \\
2.0326 & 8.2758
\end{array}\right] .
\end{aligned}
$$

Then, we have $\mathscr{A}=\check{A}_{4}+\mathscr{L} \check{A}_{2}=\left[\begin{array}{cc}0.0491 & 0.1255 \\ -0.8161 & 0.7081\end{array}\right]$ and $\mathscr{L} \check{G}_{1}+\check{G}_{2}=10^{-14} \times\left[\begin{array}{l}-0.1887 \\ -0.1332\end{array}\right]$. The Jordan canonical form of $\mathscr{A}$ is $J=\left[\begin{array}{cc}0.3 & 0 \\ 0 & 0.4571\end{array}\right]$, and then, an interval observer in the form of (30) can be designed. Figures 3-6 show that the interval state estimations through reduced-order and full-order interval observers. By comparison of the results, the reduced-order method proposed in the paper can make estimations successfully even if there is actuator fault. It is easy to know

$$
M=\left[\begin{array}{cccc}
0.2116 & 0.2116 & 0 & -0.2116 \\
0.5769 & -0.4231 & 0 & 0.4231 \\
-0.2116 & 0.7884 & 0 & 0.2116 \\
-0.2116 & -0.2116 & 1 & 0.2116
\end{array}\right] .
$$

And, the interval reconstruction of the actuator fault is given in Figure 7.

5.2. Practical System. In the second simulation, a DC motor model in [34] is considered, which has the following system dynamics: 


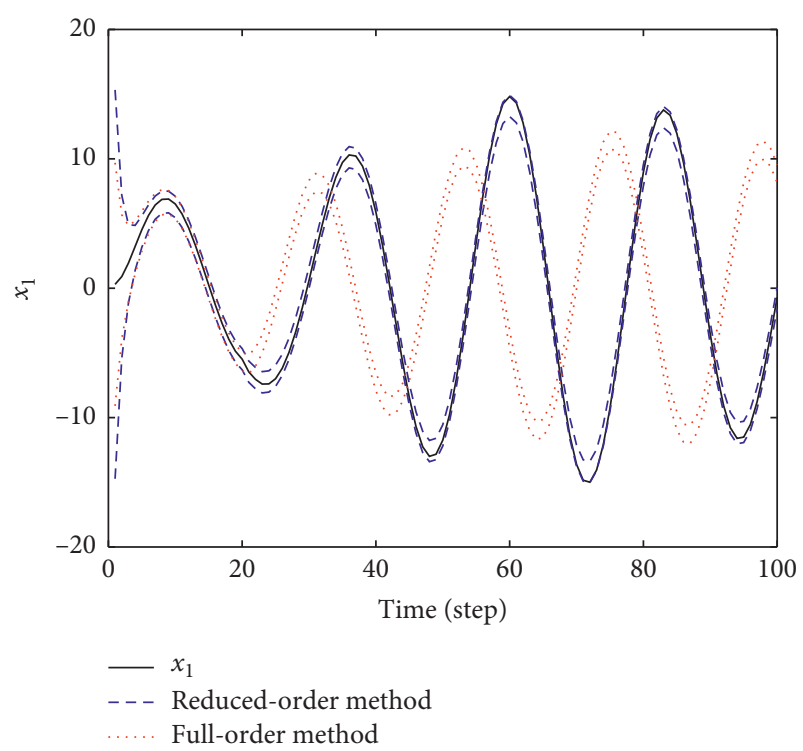

FIgURE 3: $x_{1}$ and its interval estimations with actuator faults.

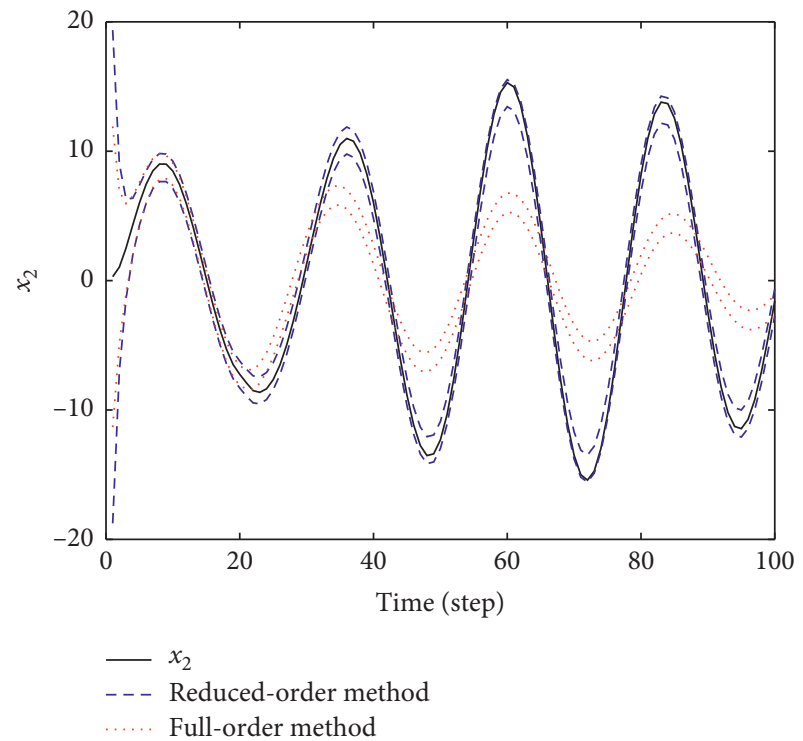

Figure 4: $x_{2}$ and its interval estimations with actuator faults.

$$
\left[\begin{array}{c}
\frac{\mathrm{d} i}{\mathrm{~d} t} \\
\frac{\mathrm{d} n}{\mathrm{~d} t}
\end{array}\right]=\left[\begin{array}{cc}
-R_{a} / L & -K_{e} / L \\
K_{t} / J_{1} & -f_{r} / J_{1}
\end{array}\right]\left[\begin{array}{c}
i \\
n
\end{array}\right]+\left[\begin{array}{c}
1 / L \\
0
\end{array}\right] u,
$$

where $n$ is the motor speed and $i$ and $u$ are the armature current and voltage, respectively. In the model, the parameters $R_{a}, L, K_{e}, K_{t}, f_{r}$, and $J_{1}$ denote the resistance, inductance, back electromotive force constant, torque constant, frictional constant, and motor inertial,

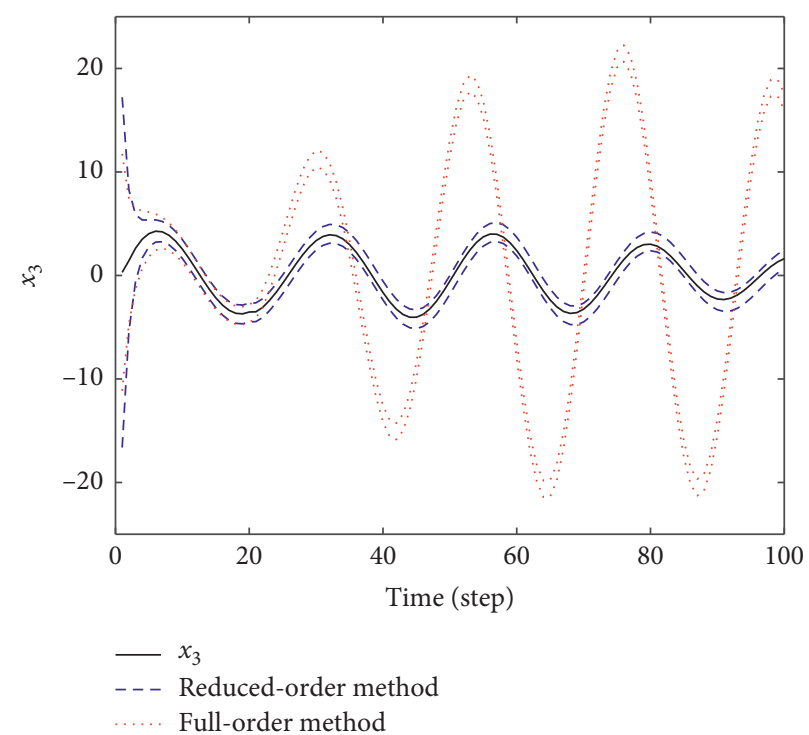

FIgURE 5: $x_{3}$ and its interval estimations with actuator faults.

respectively. The nominal parameters are given as follows: $R_{a}=1.2030(\Omega), L=5.5840 \times 10^{-3}(H), K_{e}=8.5740 \times 10^{-2}$ $(\mathrm{Vrad} / \mathrm{s}), K_{t}=8.5783 \times 10^{-2}(\mathrm{Vrad} / \mathrm{s}), \quad f_{r}=2.4500 \times 10^{-4}$ $(\mathrm{Nms} / \mathrm{rad})$, and $J_{1}=1.4166 \times 10^{-4}(\mathrm{Nms} / \mathrm{rad})$.

The system dynamics are discretized by the forward Euler method with the sampling time $T=1 \mathrm{~ms}$. We have the following system parameters:

$$
\begin{aligned}
A & =\left[\begin{array}{cc}
0.7846 & -0.0154 \\
0.6056 & 0.9983
\end{array}\right], \\
B & =\left[\begin{array}{c}
0.1791 \\
0
\end{array}\right], \\
C & =\left[\begin{array}{ll}
1 & 0 \\
0 & 1
\end{array}\right], \\
G & =\left[\begin{array}{l}
1 \\
2
\end{array}\right], \\
D & =\left[\begin{array}{l}
0.1 \\
0.1
\end{array}\right], \\
F & =\left[\begin{array}{l}
0.1 \\
0.1
\end{array}\right] .
\end{aligned}
$$

Similarly, a reduced-order interval observer can be designed for this practical system. By computing LMIs (25) and (26), we have

$$
\begin{aligned}
& P=2.5364 \times 10^{5}, \\
& Q=10^{7} \times\left[\begin{array}{ll}
-3.0690 & -2.4096
\end{array}\right], \\
& \mathscr{L}=\left[\begin{array}{ll}
-120.9991 & -94.9991
\end{array}\right] .
\end{aligned}
$$

In the simulation, the initial value of system state is $x_{0}=\left[\begin{array}{ll}0 & 0\end{array}\right]^{\mathrm{T}}$, and it is assumed that the initial values of estimated upper and lower bound are $\left[\begin{array}{ll}0.2 & 0.2\end{array}\right]^{\mathrm{T}}$ and $\left[\begin{array}{ll}-0.2 & -0.2\end{array}\right]^{\mathrm{T}}$, respectively. Besides, the control input and actuator fault are set as $u_{k}=0$ and 


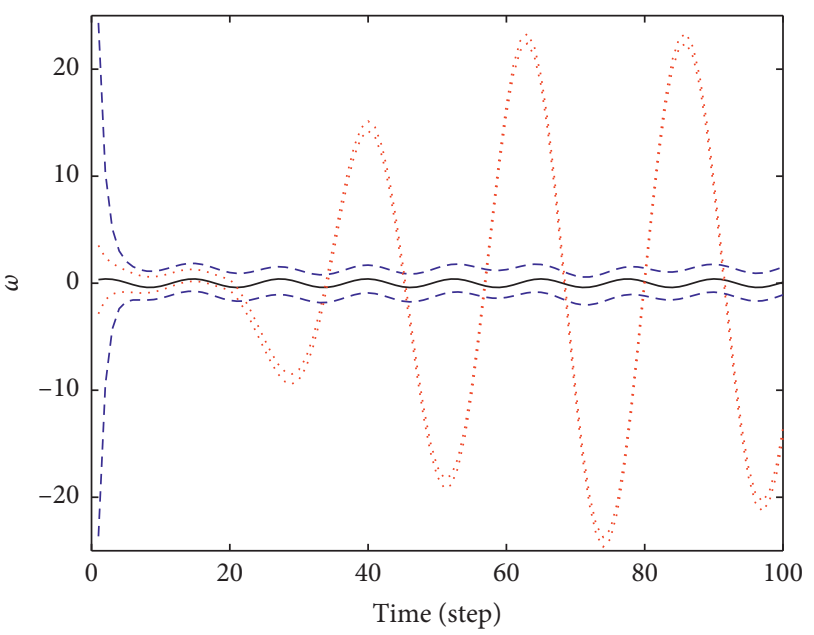

$\omega$

- - - Reduced-order method Full-order method

Figure 6: $\omega$ and its interval estimations with actuator faults.

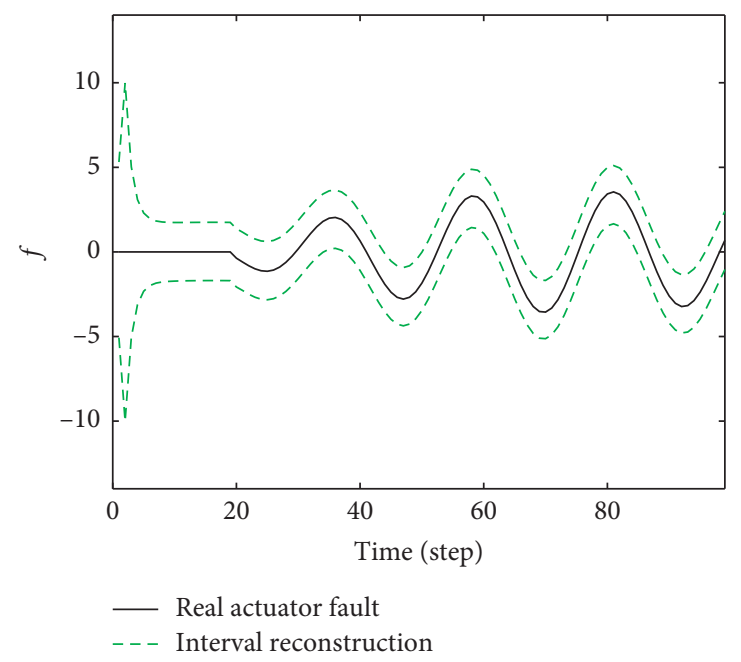

FIgURE 7: Actuator fault interval reconstruction.

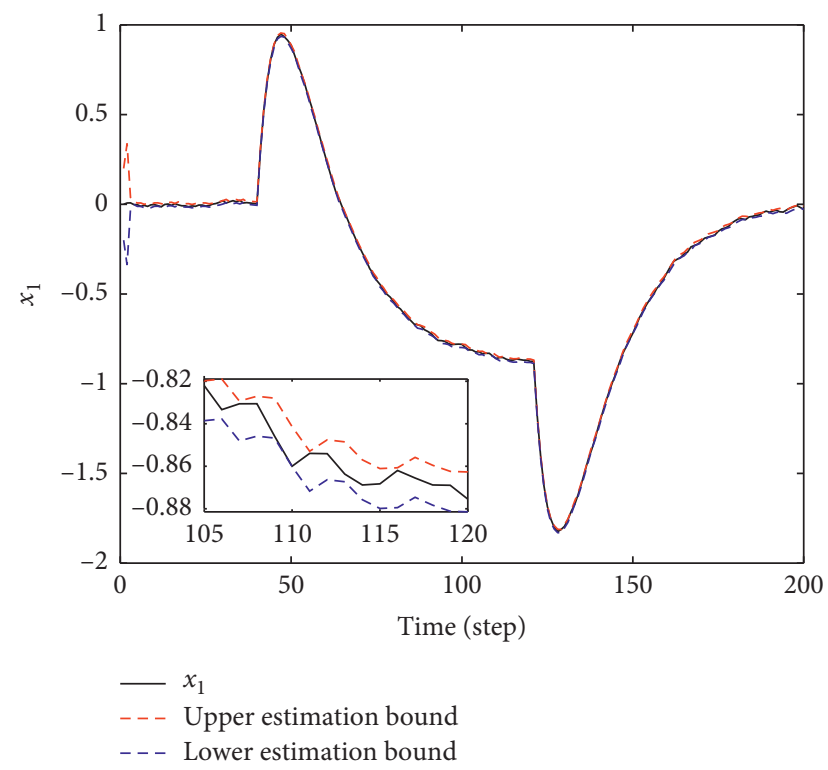

FIGURE 8: $x_{1}$ and its interval estimations with actuator faults. 


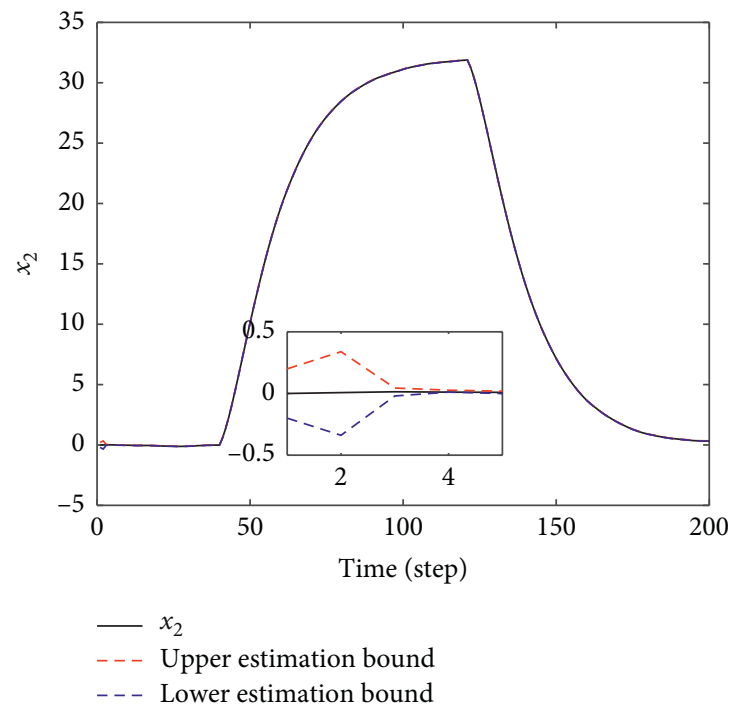

FIGURE 9: $x_{2}$ and its interval estimations with actuator faults.

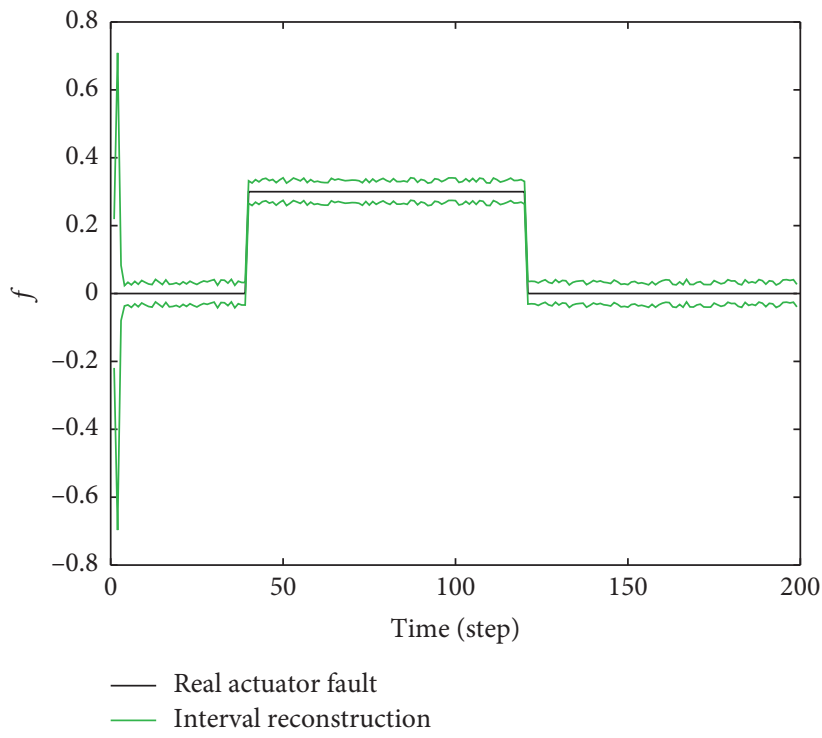

FIGURE 10: Actuator fault interval reconstruction.

$f_{k}=0.3(40 \leq k \leq 120), f_{k}=0$ (else). The unknown input and measurement noise are assumed to be stochastic and the unknown input is bounded by $|\eta| \leq 0.1$. The interval estimation of the motor speed and the armature current with the existence of actuator fault are shown in Figures 8 and 9, and the interval reconstruction of actuator fault is presented in Figure 10. The estimation results of this example verify the effectiveness and practicability of the proposed method further.

\section{Conclusion}

This paper concerns the descriptor system form used in the interval estimations. A full-order interval observer is developed as the initial actuator fault detector for linear discretetime systems when there exist actuator faults and unknown disturbances. A reduced-order one is devised by minimizing the effects of actuator faults when systems are running. Based on its estimation results, an interval reconstruction method for actuator faults is given. At last, two simulation examples illustrate the proposed full-order interval observers can serve as fault detectors and the reduced-order one can produce a reconstruction of actuator faults effectively.

\section{Data Availability}

No data were used to support this study.

\section{Conflicts of Interest}

The authors declare that there are no conflicts of interest regarding the publication of this paper. 


\section{Acknowledgments}

This research was funded by the Headquarters Science and Technology Guide Project of SGCC Grant no. 523HQ200054 and National Natural Science Foundation of China Grant no. 61304104 and also funded by Chongqing Technology Innovation and Application Special Key Project under Grant no. cstc 2019jscx-mbdxX0015.

\section{References}

[1] J. Chen and R. J. Patton, Robust Model-Based Fault Diagnosis for Dynamic Systems, Springer Science \& Business Media Press, 2nd edition, 1999.

[2] C. Edwards, S. K. Spurgeon, and R. J. Patton, "Sliding mode observers for fault detection and isolation," Automatica, vol. 36, no. 4, pp. 541-553, 2000.

[3] Z. Zhang and I. M. Jaimoukha, "On-line fault detection and isolation for linear discrete-time uncertain systems," Automatica, vol. 50, no. 2, pp. 513-518, 2014.

[4] F. Zhu and J. Yang, "Fault detection and isolation design for uncertain nonlinear systems based on full-order, reducedorder and high-order high-gain sliding-mode observers," International Journal of Control, vol. 86, no. 10, pp. 1800-1812, 2013.

[5] R. Zhang, H. Tao, L. Wu, and Y. Guan, "Transfer learning with neural networks for bearing fault diagnosis in changing working conditions," IEEE Access, vol. 5, pp. 14347-14357, 2017.

[6] B. Jiang, M. Staroswiecki, and V. Cocquempot, "Fault accommodation for nonlinear dynamic systems," IEEE Transactions on Automatic Control, vol. 51, no. 9, pp. 1578-1583, 2006.

[7] B. Kulcsar and M. Verhaegen, "Robust inversion based fault estimation for discrete-time LPV systems," IEEE Transactions on Automatic Control, vol. 57, no. 6, pp. 1581-1586, 2012.

[8] Z. Gao, C. Cecati, and S. X. Ding, "A survey of fault diagnosis and fault-tolerant techniques-part I: fault diagnosis with model-based and signal-based approaches," IEEE Transactions on Industrial Electronics, vol. 62, no. 6, pp. 3757-3767, 2015.

[9] S. Guo and F. Zhu, "Reduced-order observer design for discrete-time descriptor systems with unknown inputs: an linear matrix inequality approach," Journal of Dynamic Systems Measurement and Control, vol. 137, no. 8, Article ID 084503, 2015.

[10] K. Zhang, B. Jiang, and P. Shi, “Adjustable parameter-based distributed fault estimation observer design for multiagent systems with directed graphs," IEEE Transactions on Cybernetics, vol. 47, no. 2, pp. 306-314, 2017.

[11] Z. Gao and H. Wang, "Descriptor observer approaches for multivariable systems with measurement noises and application in fault detection and diagnosis," Systems \& Control Letters, vol. 55, no. 4, pp. 304-313, 2006.

[12] Z. Gao, "Fault estimation and fault-tolerant control for discrete-time dynamic systems," IEEE Transactions on Industrial Electronics, vol. 62, no. 6, pp. 3874-3884, 2015.

[13] B. Jiang, K. Zhang, and P. Shi, "Integrated fault estimation and accommodation design for discrete-time Takagi-Sugeno fuzzy systems with actuator faults," IEEE Transactions on Fuzzy Systems, vol. 19, no. 2, pp. 291-304, 2011.

[14] J. L. Gouzé, A. Rapaport, and M. Z. Hadj-Sadok, "Interval observers for uncertain biological systems," Ecological Modelling, vol. 133, no. 1-2, pp. 45-56, 2000.
[15] D. Efimov, T. Raïssi, S. Chebotarev, and A. Zolghadri, "Interval state observer for nonlinear time varying systems," Automatica, vol. 49, no. 1, pp. 200-205, 2013.

[16] F. Mazenc, T. N. Dinh, and S. I. Niculescu, "Interval observers for discrete-time systems," International Journal of Robust and Nonlinear Control, vol. 24, no. 17, pp. 2867-2890, 2014.

[17] M. Moisan and O. Bernard, "Robust interval observers for global Lipschitz uncertain chaotic systems," Systems \& Control Letters, vol. 59, no. 11, pp. 687-694, 2010.

[18] T. Raïssi, D. Efimov, and A. Zolghadri, "Interval state estimation for a class of nonlinear systems," IEEE Transactions on Automatic Control, vol. 57, no. 1, pp. 260-265, 2016.

[19] G. Zheng, D. Efimov, F. J. Bejarano, W. Perruquetti, and $\mathrm{H}$. Wang, "Interval observer for a class of uncertain nonlinear singular systems," Automatica, vol. 71, no. C, pp. 159-168, 2016.

[20] L. Meyer, D. Ichalal, and V. Vigneron, "Interval observer for LPV systems with unknown inputs," IET Control Theory \& Applications, vol. 12, no. 5, pp. 649-660, 2018.

[21] Y. Wang, D. M. Bevly, and R. Rajamani, "Interval observer design for LPV systems with parametric uncertainty," Automatica, vol. 60, no. 1, pp. 79-85, 2015.

[22] S. Guo, B. Jiang, F. Zhu, and Z. Wang, "Luenberger-like interval observer design for discrete-time descriptor linear system," Systems \& Control Letters, vol. 126, pp. 21-27, 2019.

[23] W. Ren, R. You, W. Yu, and S. Guo, "Reduced-order and fullorder interval observers design for discrete-time system," IEEE Access, vol. 8, pp. 103309-103316, 2020.

[24] Z. Wang, Y. Shen, and X. Zhang, "Actuator fault estimation for a class of nonlinear descriptor systems," International Journal of Systems Science, vol. 45, no. 3, pp. 487-496, 2014.

[25] J. Meseguer, V. Puig, T. Escobet, and J. Saludes, "Observer gain effect in linear interval observer-based fault detection," Journal of Process Control, vol. 20, no. 8, pp. 944-956, 2010.

[26] C. Edwards, S. K. Spurgeon, and R. J. Patton, "A note on interval observer design for unknown input estimation," International Journal of Control, vol. 89, no. 1, pp. 541-553, 2016.

[27] C. Edwards, S. K. Spurgeon, and R. J. Patton, "Fault detection based on robust adaptive thresholds: a dynamic interval approach," Annual Reviews in Control, vol. 37, no. 1, pp. 541-553, 2013.

[28] S. Guo and F. Zhu, "Actuator fault detection and interval reconstruction based on interval observers," in Proceedings Conference 20th IFAC World Congress, pp. 1-8, Toulouse, France, July 2017.

[29] X. Zhang and F. Zhu, "Observer-based sensor attack diagnosis for cyber-physical systems via zonotope theory," Asian Journal of Control, vol. 60, 2020.

[30] Z. Zhang and G. Yang, "Distributed fault detection and isolation for multiagent systems: an interval observer approach," IEEE Transactions on Systems, Man, and Cybernetics, vol. 50, no. 6, pp. 2220-2230, 2018.

[31] M. Pourasghar, V. Puig, and C. Ocampo-Martinez, "Characterisation of interval-observer fault detection and isolation properties using the set-invariance approach," Journal of the Franklin Institute, vol. 357, no. 3, pp. 1853-1886, 2020.

[32] F. Zhu, Y. Tang, and Z. Wang, "Interval-Observer-based fault detection and isolation design for T-S fuzzy system based on zonotope analysis," IEEE Transactions on Fuzzy Systems, vol. 40, p. 1, 2021.

[33] L. Farina and S. Rinaldi, Positive Linear Systems: Theory and Applications, Wiley, Hoboken, NJ, USA, 2011.

[34] W. Tang, Z. Wang, and Y. Shen, "Interval estimation for discrete-time linear systems: a two-step method," Systems \& Control Letters, vol. 123, pp. 69-74, 2019. 